1 Fundação Oswaldo Cruz (Fiocruz), Escola Nacional de Saúde Pública Sergio Arouca (Ensp) - Rio de Janeiro (RJ), Brasil. Orcid: https://orcid org/0000-0002-37129217

deniclara.brocardo@gmail. com

2 Fundação Oswaldo Cruz (Fiocruz), Escola Naciona de Saúde Pública Sergio Arouca (Ensp) - Rio de Janeiro (RJ), Brasil. Orcid: https://orcid org/0000-0003-3232

0917

carlamv@ensp.fiocruz.br

3 Fundação Oswaldo Cruz (Fiocruz), Escola Nacional de Saúde Pública Sergio Arouca (Ensp) - Rio de Janeiro (RJ), Brasil. Orcid: https://orcid org/0000-0003-07463684

marciafausto@ensp.fiocruz.br

4 Fundação Oswaldo Cruz (Fiocruz), Escola Nacional de Saúde Pública Sergio Arouca (Ensp) - Rio de Janeiro (RJ), Brasil. Orcid: https://orcid. org/0000-0003-14500498

slemos@ensp.fiocruz.br

\section{Núcleo de Apoio à Saúde da Família (Nasf): panorama nacional a partir de dados do PMAQ}

\author{
Family Health Support Center (Nasf): a national panorama based on \\ National Program for Access and Quality Improvement (PMAQ) data
}

Deniclara Brocardo $\mathbf{1}$, Carla Lourenço Tavares de Andrade ${ }^{2}$, Márcia Cristina Rodrigues Fausto ${ }^{\mathbf{3}}$, Sheyla Maria Lemos Lima4

DOI: 10.1590/0103-110420185109

RESUMO O Núcleo de Apoio à Saúde da Família (Nasf) foi criado em 2008 visando aumentar a resolutividade e o escopo das ações da Atenção Básica (AB). Composto por uma equipe multiprofissional deve desenvolver atividades conjuntas com as equipes $\mathrm{AB}$, seguindo a lógica do apoio matricial e das ações técnico-pedagógicas ou clínico-assistenciais, pretendendo a integralidade do cuidado. O objetivo deste estudo foi analisar o trabalho do Nasf no território brasileiro, considerando a integração entre as equipes Nasf e $\mathrm{AB}$, a partir de dados provenientes da avaliação externa do Programa Nacional para Melhoria do Acesso e da Qualidade (PMAQ) segundo ciclo. Os resultados encontrados apontam adequação quanto à infraestrutu$\mathrm{ra}$, às atividades de apoio matricial e às atividades integradas com as equipes $\mathrm{AB}$. Contudo, o monitoramento e análise de indicadores, a formação inicial e a educação permanente carecem de maior desenvolvimento.

PALAVRAS-CHAVE Atenção Primária à Saúde. Avaliação em saúde. Saúde da família. Política de saúde.

ABSTRACT The Family Health Support Center (Nasf) was created in 2008 and aims to increase the resolution and scope of Primary Care $(A B)$ actions. Composed by a multiprofessional team, it should develop joint activities with the $A B$ teams, following the logic of matrix support and technical-pedagogical or clinical-assistancial actions, aiming at the integrality of care. The objective of this study was to analyze the work of the Nasf in Brazilian territory, considering the integration between the Nasf and $A B$ teams, based on data from the external evaluation of the National Program for Access and Quality Improvement (PMAQ) in the second cycle. The results found show adequacy of the infrastructure, matrix support activities and activities integrated with the $A B$ teams. However, the monitoring and analysis of indicators, the initial training and the continuing education need greater attention.

KEYWORDS Primary Health Care. Health evaluation. Family health. Health policy. 


\section{Introdução}

A Atenção Primária a Saúde (APS) no Brasil, também denominada de Atenção Básica (AB), tem assumido em muitos países papel de relevância na organização do sistema público de saúde. Vários estudos internacionais e nacionais apontam resultados positivos a partir de sua atuação, referindo maior acesso aos serviços, melhor qualidade dos cuidados, maior foco na prevenção e redução de cuidados especializados desnecessários. Considera-se que uma APS resolutiva possa contribuir para maior equidade no sistema de saúde em geral, para a prestação de atenção em saúde mais eficaz e para reduzir a mortalidade por mortes prematuras, doenças respiratórias e cardiovasculares ${ }^{\mathbf{1 - 4}}$.

Embora haja relativo consenso sobre a importância da APS na organização do sistema de saúde, diferentes arranjos nas práticas realizadas e na composição de profissionais envolvidos na APS são registrados ${ }^{4-6}$.

O médico generalista é o profissional mais comum em diferentes arranjos organizacionais. Contudo, observa-se, em muitos países, uma tendência à composição de equipes multiprofissionais para potencializar a cooperação interdisciplinar e aumentar a capacidade resolutiva da APS $^{7-9}$.

No Brasil, desde o final dos anos de 1990, o Ministério da Saúde (MS) tem investido e induzido a saúde da família como modelo para organização das práticas da APS. A Estratégia Saúde da Família (ESF) tem como núcleo organizador do trabalho uma equipe mínima composta por médico, enfermeiro, técnico de enfermagem e agentes comunitários de saúde, responsáveis por uma população de aproximadamente 3 mil pessoas cadastradas à equipe, em territórios definidos. As equipes ESF podem estar ligadas a uma equipe de Saúde Bucal (odontólogo, auxiliar e/ou técnico em saúde bucal) e a um Núcleo de Apoio à Saúde da Família (Nasf) ${ }^{\mathbf{1 0}}$.

O Nasf, criado, em 2008, com o objetivo de qualificar e tornar mais resolutiva a atuação da $\mathrm{AB}$, é constituído por uma equipe multiprofissional. A composição da equipe
Nasf, segundo diretriz ministerial, deve ser definida pelo gestor municipal, em consulta às equipes $\mathrm{AB}$, de acordo com as necessidades identificadas no território, com o perfil epidemiológico e com as condições socioeconômicas da população ${ }^{11}$. Espera-se que a atuação do Nasf seja de forma integrada e colaborativa com as equipes $\mathrm{AB}$, segundo a lógica do apoio matricial, que envolve a atuação conjunta das duas equipes, em atividades de natureza técnico-pedagógica e clínico-assistencial ${ }^{12,13}$. Embora apresentadas de forma separada, essas dimensões estão, na prática, fortemente imbricadas. Em tese, a atuação matricial incrementaria a resolutividade e a integralidade da atenção prestada pela $\mathrm{AB}$.

Embora se identifique a potencialidade do Nasf na resolução e qualificação da $\mathrm{AB}^{14-16}$, ainda são identificadas diferentes formas de implantação e operacionalização do Nasf; e é relatada pouca clareza dos profissionais quanto ao modo de operar o apoio matricial16-18.

Compreender a dinâmica do funcionamento das equipes Nasf na sua interface com as equipes $\mathrm{AB}$ pode ajudar na identificação de ações que contribuam para a operacionalização da atuação compartilhada entre as duas equipes. O instrumento de avaliação do Programa Nacional para Melhoria do Acesso e da Qualidade (PMAQ), entre outros aspectos, possibilita caracterizar as atividades desenvolvidas de forma conjunta entre equipes Nasf e AB no campo da gestão, planejamento, avaliação e no campo da assistência propriamente dita.

Dessa forma, este artigo objetiva caracterizar o desenvolvimento das atividades que compõem a atuação matricial do Nasf em todo território nacional, a partir da avaliação externa do $2^{\circ}$ ciclo do PMAQ.

\section{Material e métodos}

Trata-se de um estudo descritivo, transversal, baseado em dados secundários do PMAQ do $2^{\circ}$ ciclo da avaliação externa, relativo ao 
período 2013-2014. Os dados utilizados são de domínio público e de acesso irrestrito, disponibilizados pelo Departamento de Atenção Básica (DAB) do MS ${ }^{19}$.

O PMAQ é um programa de avaliação e monitoramento da $\mathrm{AB}$ do $\mathrm{MS}$ composto por quatro fases. A avaliação externa é a terceira fase, na qual são observadas, in loco, as condições de funcionamento das equipes que aderiram ao programa. É realizada por meio de parceria entre o MS e Instituições de Ensino e Pesquisa, que ficam responsáveis pela coleta de dados em todo o território nacional. O instrumento de avaliação externa, aplicado por pesquisadores capacitados, é composto por seis módulos: o primeiro e o quinto dispõem sobre a infraestrutura das Unidades Básicas de Saúde (UBS). O segundo, quarto e sexto módulos são direcionados aos profissionais da equipe $A B$, equipe Nasf e equipe de Saúde Bucal, respectivamente. O terceiro módulo é aplicado aos usuários.

Para o presente estudo, foram consideradas, das 91 variáveis do Modulo IV, apenas aquelas que contemplavam a caracterização da estrutura física disponível, oferta de capacitação, ações de gestão, planejamento, coordenação das equipes e de desenvolvimento do apoio matricial.

Os dados foram analisados por meio de estatística descritiva; e as tabulações, frequências simples, porcentagem e cruzamentos foram realizados por meio do software SPSS (Statistical Package for the Social Sciences), versão 20.

A pesquisa foi submetida ao Comitê de Ética em Pesquisa da Ensp/Fiocruz no mês de maio/2017, identificado pelo CAAE 68410017.3.0000.5240, sendo aprovada pelo parecer consubstanciado de número 2.111.925, em 9 de junho de 2017.

\section{Resultados e discussão}

\section{Adesão ao PMAQ}

Foram consideradas 1.774 equipes Nasf que responderam à avaliação externa do $2^{\circ}$ ciclo do PMAQ, relativo ao período 2013-2014, correspondendo a $45,5 \%$ das equipes Nasf informadas pelo MS em 2014. O baixo percentual registrado de participação das equipes Nasf contrastou com os 75,8\% de participação das equipes $\mathrm{AB}$ (tabela 1). A participação das equipes Nasf e AB no PMAQ perante o número de equipes informadas pelo MS evidenciou uma situação relativamente semelhante entre as regiões do País, porém bastante diversa entre os estados.

Considerando as equipes Nasf, as regiões Sudeste e Nordeste apresentaram percentuais de adesão pouco maiores que a média referida, $50,0 \%$ e $47,5 \%$, respectivamente. A região Sul registrou o menor percentual, $35,3 \%$. Destoaram os estados do Tocantins (19,4\%), do Espírito Santo (25,0\%), do Maranhão (28,6\%) e do Rio Grande do Sul $(29,5 \%)$, com as menores participações. Com elevada participação, destacaram-se os estados do Amapá com 85,0\% e os de Rondônia, do Ceará, de Pernambuco, do Rio de Janeiro e de Mato Grosso do Sul, todos com mais de $60,0 \%$ das equipes Nasf participando do $2^{\circ}$ ciclo do PMAQ (tabela 1 ). 
Tabela 1. Distribuição das equipes $A B$ e Nasf informadas pelo Ministério da Saúde e respondentes da avaliação externa do $2^{\circ}$ ciclo do $P M A Q$, segundo Grandes Regiões e Unidades da Federação, 2014

\begin{tabular}{|c|c|c|c|c|c|c|c|c|}
\hline \multirow[b]{2}{*}{$\begin{array}{l}\text { Grandes Regi- } \\
\text { ões e Unidades } \\
\text { da Federação }\end{array}$} & \multicolumn{3}{|c|}{ Equipes $\mathrm{AB}$} & \multicolumn{3}{|c|}{ Equipes Nasf } & \multirow[b]{2}{*}{$\begin{array}{r}\text { Número de } \\
\text { equipes } A B \\
\text { com Nasf } \\
\text { referido }^{\star \star}\end{array}$} & \multirow[b]{2}{*}{$\begin{array}{r}\text { N Equipes } A B \\
\text { implantadas / } \\
\text { N Equipes Nasf } \\
\text { implantadas }\end{array}$} \\
\hline & $\begin{array}{r}\text { N implanta- } \\
\text { das }^{\star}\end{array}$ & 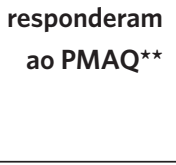 & $\begin{array}{r}\% \mathrm{AB} \text { implan- } \\
\text { tada que res- } \\
\text { ponderam ao } \\
\mathrm{PMAQ}\end{array}$ & $\begin{array}{r}\text { N implanta- } \\
\text { das }^{\star}\end{array}$ & $\begin{array}{l}\text { V responderam } \\
\text { ao } \mathrm{PMAQ}^{\star \star}\end{array}$ & $\begin{array}{r}\% \text { Nasf im- } \\
\text { plantadas que } \\
\text { responderam } \\
\text { ao PMAQ }\end{array}$ & & \\
\hline Brasil & 39.310 & 29.778 & 75,8 & 3.898 & 1.774 & 45,5 & 17.157 & 10,1 \\
\hline Norte & 3.189 & 2.160 & 67,7 & 312 & 126 & 40,4 & 997 & 10,2 \\
\hline Rondônia & 368 & 252 & 68,5 & 14 & 9 & 64,3 & 109 & 26,3 \\
\hline Acre & 209 & 99 & 47,4 & 21 & 9 & 42,9 & 42 & 10,0 \\
\hline Amazonas & 671 & 469 & 69,9 & 55 & 29 & 52,7 & 164 & 12,2 \\
\hline Roraima & 115 & 77 & 67,0 & 9 & 4 & 44,4 & 20 & 12,8 \\
\hline Pará & 1.196 & 776 & 64,9 & 90 & 38 & 42,2 & 368 & 13,3 \\
\hline Amapá & 160 & 126 & 78,8 & 20 & 17 & 85,0 & 105 & 8,0 \\
\hline Tocantins & 470 & 361 & 76,8 & 103 & 20 & 19,4 & 189 & 4,6 \\
\hline Nordeste & 14.836 & 10.768 & 72,6 & 1.730 & 821 & 47,5 & 7.420 & 8,6 \\
\hline Maranhão & 2.004 & 660 & 32,9 & 154 & 44 & 28,6 & 342 & 13,0 \\
\hline Piauí & 1.266 & 865 & 68,3 & 234 & 86 & 36,8 & 696 & 5,4 \\
\hline Ceará & 2.303 & 1.626 & 70,6 & 215 & 141 & 65,6 & 1.315 & 10,7 \\
\hline $\begin{array}{l}\text { Rio Grande do } \\
\text { Norte }\end{array}$ & 1.018 & 862 & 84,7 & 160 & 57 & 35,6 & 616 & 6,4 \\
\hline Paraíba & 1.344 & 1.211 & 90,1 & 262 & 116 & 44,3 & 979 & 5,1 \\
\hline Pernambuco & 2.142 & 1.845 & 86,1 & 218 & 141 & 64,7 & 1.322 & 9,8 \\
\hline Alagoas & 820 & 698 & 85,1 & 125 & 54 & 43,2 & 469 & 6,6 \\
\hline Sergipe & 634 & 376 & 59,3 & 33 & 12 & 36,4 & 148 & 19,2 \\
\hline Bahia & 3.305 & 2.625 & 79,4 & 329 & 170 & 51,7 & 1.533 & 10,0 \\
\hline Sudeste & 12.934 & 10.100 & 78,1 & 1.043 & 521 & 50,0 & 5.575 & 12,4 \\
\hline Minas Gerais & 5.079 & 4.180 & 82,3 & 629 & 295 & 46,9 & 2.558 & 8,1 \\
\hline Espírito Santo & 698 & 516 & 73,9 & 20 & 5 & 25,0 & 58 & 34,9 \\
\hline Rio de Janeiro & 2.377 & 1.881 & 79,1 & 117 & 71 & 60,7 & 1.047 & 20,3 \\
\hline São Paulo & 4.780 & 3.523 & 73,7 & 277 & 150 & 54,2 & 1.912 & 17,3 \\
\hline Sul & 5.620 & 4.509 & 80,2 & 535 & 189 & 35,3 & 2.070 & 10,5 \\
\hline Paraná & 2.209 & 1.789 & 81,0 & 209 & 76 & 36,4 & 817 & 10,6 \\
\hline Santa Catarina & 1.642 & 1.467 & 89,3 & 221 & 82 & 37,1 & 916 & 7,4 \\
\hline $\begin{array}{l}\text { Rio Grande } \\
\text { do Sul }\end{array}$ & 1.769 & 1.253 & 70,8 & 105 & 31 & 29,5 & 337 & 16,8 \\
\hline Centro-Oeste & 2.731 & 2.241 & 82,1 & 278 & 117 & 42,1 & 1.095 & 9,8 \\
\hline $\begin{array}{l}\text { Mato Grosso } \\
\text { do Sul }\end{array}$ & 546 & 437 & 80,0 & 60 & 38 & 63,3 & 296 & 9,1 \\
\hline Mato Grosso & 630 & 514 & 81,6 & 44 & 17 & 38,6 & 192 & 14,3 \\
\hline Goiás & 1.313 & 1.180 & 89,9 & 168 & 60 & 35,7 & 550 & 7,8 \\
\hline $\begin{array}{l}\text { Distrito Fe- } \\
\text { deral }\end{array}$ & 242 & 110 & 45,5 & 6 & 2 & 33,3 & 57 & 40,3 \\
\hline
\end{tabular}

Fonte: Elaboração própria, com base em dados do Ministério da Saúde 20.

*Ministério da Saúde, 2017.

**Banco de dados PMAQ, segundo ciclo, 2014 
Quanto à participação das equipes $\mathrm{AB}$, a região Norte registrou a menor (67,7\%); e a Centro-Oeste, a maior participação (82,1\%). As menores participações foram observadas no Maranhão (32,9\%), no Distrito Federal (45,5\%) e no Acre (47,4\%). O estado com maior participação foi o da Paraíba (90,1\%). Nos estados do Rio Grande do Norte, de Pernambuco, de Alagoas, de Minas Gerais, do Paraná, de Santa Catarina, de Mato Grosso, do Mato Grosso do Sul e de Goiás, ao menos $80,0 \%$ das equipes $\mathrm{AB}$ participaram do PMAQ (tabela 1).

A diferença entre a participação das equipes $\mathrm{AB}$ e Nasf no PMAQ, em parte, pode ser explicada por ter sido a primeira vez que o Nasf participou da avaliação do PMAQ, diferentemente da equipe $A B$, que participa desde o início do processo. Além disso, a participação da equipe Nasf no PMAQ não é uma decisão da equipe, e, sim, do gestor municipal juntamente com a equipe $A B$ que, de forma voluntária, aderem ao PMAQ. A participação do Nasf está condicionada à adesão da equipe $A B$ à qual está vinculada $\mathbf{2 1 , 2 2}^{2}$

Por outro lado, as diferenças observadas na participação das equipes Nasf e $A B$ no PMAQ entre os estados podem, em parte, ser explicadas pela heterogeneidade decorrente da descentralização de saúde e do federalismo fiscal que provocam iniquidades na oferta e acesso aos serviços de saúde ${ }^{23}$.

Outro aspecto importante a ser observado refere-se à disparidade da razão entre equipes $\mathrm{AB}$ e Nasf implantadas entre os estados do País. Observou-se que o Tocantins $(4,6)$, o Piauí $(5,4)$ e a Paraíba $(5,1)$ apresentaram as menores razões, enquanto o Distrito Federal $(40,3)$, o Espírito Santo $(34,9)$ e Rondônia $(26,3)$, os valores mais elevados ao se dividir o número de equipes $\mathrm{AB}$ pelo número de equipes Nasf (tabela 1).

Considerando-se que as diretrizes ministeriais preconizam, no máximo, nove equipes
AB por equipe Nasf (dependendo do tipo de Nasf), supõe-se que os estados que apresentaram razão menor ou próxima ao padrão seriam aqueles com melhores resultados de implantação do Nasf. Em contrapartida, destacaram-se negativamente os estados com razões muito elevadas, indicando uma implantação do Nasf ainda incipiente ${ }^{24}$.

De toda forma, houve um incremento na implantação dos Nasf no País. Considerando a razão entre equipes $\mathrm{AB}$ e Nasf, em estudo realizado em 2012, observou-se que as regiões Norte $(16,9)$, Nordeste $(18,1)$, Sudeste $(21,1)$, Sul $(33,4)$ e Centro-Oeste $(20,9)$ apresentaram razões bem superiores (16). Enquanto as encontradas no estudo atual foram, respectivamente, 10,2, 8,6, 12,4, 10,5 e 9,8.

Nos resultados a seguir apresentados, sintetizados nas tabelas 2, 3, 4 e 5, o total de equipes respondentes (N) é diferente entre si para algumas questões e diferente do total de equipes que participaram do PMAQ (1.774 equipes Nasf), pois nem sempre todas as questões foram respondidas por todas as equipes. Ou seja, há diferentes $\mathrm{N}$, para as diferentes questões do instrumento de avaliação.

\section{Estrutura física e capacitação profissional}

Considerando o espaço físico utilizado para realização das atividades do Nasf, predominou a existência de consultórios compartilhados entre as equipes Nasf e $\mathrm{AB}$, assim referido por $85,7 \%$ das equipes Nasf. Ainda vale registrar que $72,8 \%$ e $82,7 \%$ das equipes, respectivamente, informaram haver sala de reuniões na unidade e espaços no território para atuação do Nasf. Por fim, destaca-se que $80,0 \%$ das equipes registraram disponibilidade de veículo, sempre ou na maioria das vezes, para realização de seu trabalho (tabela 2). 
Tabela 2. Espaços utilizados, disponibilidade de veículo, formação específica e educação permanente para as equipes Nasf, segundo Grandes Regiões e Unidades da Federação, 2014

\begin{tabular}{|c|c|c|c|c|c|c|c|c|c|c|c|c|c|c|}
\hline \multirow{3}{*}{$\begin{array}{l}\text { Grandes } \\
\text { Regiões e } \\
\text { Unidades da } \\
\text { Federação }\end{array}$} & \multicolumn{8}{|c|}{$\begin{array}{l}\text { Espaços utilizados } \\
\qquad(\mathrm{N}=1.774)\end{array}$} & \multirow{2}{*}{\multicolumn{2}{|c|}{$\begin{array}{c}\begin{array}{c}\text { Disponibilidade } \\
\text { de veículo } \\
(\mathrm{N}=1.333)\end{array} \\
\begin{array}{c}\text { Sempre } / \mathrm{Na} \\
\text { maioria das vezes }\end{array} \\
\end{array}$}} & \multirow{2}{*}{\multicolumn{2}{|c|}{\begin{tabular}{|l|}
$\begin{array}{l}\text { Formação } \\
\text { específica } \\
(\mathrm{N}=1.774)\end{array}$ \\
Para todos os \\
profissionais \\
\end{tabular}}} & \multirow{2}{*}{\multicolumn{2}{|c|}{$\begin{array}{l}\begin{array}{c}\text { Educação } \\
\text { permanente } \\
(\mathrm{N}=1.774)\end{array} \\
\text { Para todos os } \\
\text { profissionais } \\
\end{array}$}} \\
\hline & \multicolumn{2}{|c|}{$\begin{array}{r}\text { Consultório } \\
\text { exclusivo Nasf }\end{array}$} & \multicolumn{2}{|c|}{$\begin{array}{r}\text { Consultório } \\
\text { compartilhado }\end{array}$} & \multicolumn{2}{|c|}{$\begin{array}{r}\text { Sala de reuniões } \\
\text { na unidade }\end{array}$} & \multicolumn{2}{|c|}{$\begin{array}{r}\text { Espaços no } \\
\text { território } \\
\end{array}$} & & & & & & \\
\hline & $\mathrm{N}$ & $\%$ & $\mathrm{~N}$ & $\%$ & $\mathbf{N}$ & $\%$ & $\mathrm{~N}$ & $\%$ & $\mathrm{~N}$ & $\%$ & $\mathrm{~N}$ & $\%$ & $\mathrm{~N}$ & $\%$ \\
\hline Brasil & 690 & 38,9 & 1521 & 85,7 & 1291 & 72,8 & 1467 & 82,7 & 1.067 & 80,0 & 809 & 45,6 & 916 & 51,6 \\
\hline Norte & 58 & 46,0 & 93 & 73,8 & 54 & 42,9 & 77 & 61,1 & 59 & 77,6 & 38 & 30,2 & 27 & 21,4 \\
\hline Rondônia & 4 & 44,4 & 7 & 77,8 & 4 & 44,4 & 5 & 55,6 & 5 & 83,3 & - & - & - & - \\
\hline Acre & 6 & 66,7 & 4 & 44,4 & 6 & 66,7 & 5 & 55,6 & 4 & 80,0 & 2 & 22,2 & 2 & 22,2 \\
\hline Amazonas & 9 & 31,0 & 25 & 86,2 & 13 & 44,8 & 22 & 75,9 & 13 & 72,2 & 8 & 27,6 & 7 & 24,1 \\
\hline Roraima & 1 & 25,0 & 4 & 100,0 & 2 & 50,0 & - & - & - & - & - & - & - & - \\
\hline Pará & 17 & 44,7 & 28 & 73,7 & 13 & 34,2 & 19 & 50,0 & 23 & 85,2 & 16 & 42,1 & 10 & 26,3 \\
\hline Amapá & 8 & 47,1 & 13 & 76,5 & 8 & 47,1 & 12 & 70,6 & 4 & 80,0 & 6 & 35,3 & 3 & 17,6 \\
\hline Tocantins & 13 & 65,0 & 12 & 60,0 & 8 & 40,0 & 14 & 70,0 & 10 & 71,4 & 6 & 30,0 & 5 & 25,0 \\
\hline Nordeste & 311 & 37,9 & 692 & 84,3 & 570 & 69,4 & 680 & 82,8 & 572 & 82,7 & 361 & 44,0 & 385 & 46,9 \\
\hline Maranhão & 27 & 61,4 & 33 & 75,0 & 29 & 65,9 & 36 & 81,8 & 26 & 76,5 & 9 & 20,5 & 18 & 40,9 \\
\hline Piauí & 46 & 53,5 & 62 & 72,1 & 42 & 48,8 & 62 & 72,1 & 74 & 90,2 & 40 & 46,5 & 35 & 40,7 \\
\hline Ceará & 77 & 54,6 & 120 & 85,1 & 102 & 72,3 & 121 & 85,8 & 111 & 89,5 & 68 & 48,2 & 73 & 51,8 \\
\hline $\begin{array}{l}\text { Rio Grande do } \\
\text { Norte }\end{array}$ & 29 & 50,9 & 49 & 86,0 & 45 & 78,9 & 52 & 91,2 & 38 & 86,4 & 30 & 52,6 & 28 & 49,1 \\
\hline Paraíba & 32 & 27,6 & 100 & 86,2 & 84 & 72,4 & 104 & 89,7 & 71 & 82,6 & 41 & 35,3 & 52 & 44,8 \\
\hline Pernambuco & 36 & 25,5 & 124 & 87,9 & 91 & 64,5 & 110 & 78,0 & 81 & 71,7 & 68 & 48,2 & 71 & 50,4 \\
\hline Alagoas & 17 & 31,5 & 36 & 66,7 & 28 & 51,9 & 38 & 70,4 & 39 & 79,6 & 16 & 29,6 & 18 & 33,3 \\
\hline Sergipe & 8 & 66,7 & 9 & 75,0 & 10 & 83,3 & 9 & 75,0 & 10 & 83,3 & 4 & 33,3 & 6 & 50,0 \\
\hline Bahia & 39 & 22,9 & 159 & 93,5 & 139 & 81,8 & 148 & 87,1 & 122 & 82,4 & 85 & 50,0 & 84 & 49,4 \\
\hline Sudeste & 202 & 38,8 & 475 & 91,2 & 424 & 81,4 & 457 & 87,7 & 248 & 78,2 & 276 & 53,0 & 326 & 62,6 \\
\hline Minas Gerais & 136 & 46,1 & 270 & 91,5 & 246 & 83,4 & 255 & 86,4 & 190 & 80,9 & 146 & 49,5 & 164 & 55,6 \\
\hline Espírito Santo & 1 & 20,0 & 4 & 80,0 & 3 & 60,0 & 4 & 80,0 & 1 & 20,0 & 1 & 20,0 & - & - \\
\hline Rio de Janeiro & 13 & 18,3 & 68 & 95,8 & 42 & 59,2 & 67 & 94,4 & 21 & 65,6 & 37 & 52,1 & 44 & 62,0 \\
\hline São Paulo & 52 & 34,7 & 133 & 88,7 & 133 & 88,7 & 131 & 87,3 & 36 & 80,0 & 92 & 61,3 & 118 & 78,7 \\
\hline Sul & 73 & 38,6 & 170 & 89,9 & 156 & 82,5 & 163 & 86,2 & 117 & 77,0 & 82 & 43,4 & 125 & 66,1 \\
\hline Paraná & 21 & 27,6 & 67 & 88,2 & 58 & 76,3 & 62 & 81,6 & 41 & 78,8 & 42 & 55,3 & 44 & 57,9 \\
\hline Santa Catarina & 42 & 51,2 & 77 & 93,9 & 72 & 87,8 & 76 & 92,7 & 59 & 74,7 & 34 & 41,5 & 61 & 74,4 \\
\hline $\begin{array}{l}\text { Rio Grande } \\
\text { do Sul }\end{array}$ & 10 & 32,3 & 26 & 83,9 & 26 & 83,9 & 25 & 80,6 & 17 & 81,0 & 6 & 19,4 & 20 & 64,5 \\
\hline Centro-Oeste & 46 & 39,3 & 91 & 77,8 & 87 & 74,4 & 90 & 76,9 & 71 & 74,0 & 52 & 44,4 & 53 & 45,3 \\
\hline $\begin{array}{l}\text { Mato Grosso } \\
\text { do Sul }\end{array}$ & 13 & 34,2 & 30 & 78,9 & 32 & 84,2 & 28 & 73,7 & 21 & 65,6 & 17 & 44,7 & 26 & 68,4 \\
\hline Mato Grosso & 6 & 35,3 & 12 & 70,6 & 11 & 64,7 & 10 & 58,8 & 9 & 75,0 & 2 & 11,8 & 3 & 17,6 \\
\hline Goiás & 27 & 45,0 & 47 & 78,3 & 42 & 70,0 & 50 & 83,3 & 40 & 78,4 & 32 & 53,3 & 23 & 38,3 \\
\hline $\begin{array}{l}\text { Distrito Fe- } \\
\text { deral }\end{array}$ & - & - & 2 & 100,0 & 2 & 100,0 & 2 & 100,0 & 1 & 100,0 & 1 & 50,0 & 1 & 50,0 \\
\hline
\end{tabular}

Fonte: Elaboração própria, com base em dados Banco de dados PMAQ, segundo ciclo, 2014.

Nota: Dado numérico igual a zero não resultante de arredondamento. 
Os resultados foram bastante satisfatórios diante do incentivo à atuação conjunta das duas equipes, Nasf e $\mathrm{AB}$, na utilização de espaços de atendimento comuns e na inserção das equipes nos locais de moradia da população, inclusive, para tanto, com veículo disponível para seu deslocamento.

Exceções foram observadas nos estados do Acre (66,7\%), de Sergipe (66,7\%), do Tocantins (65,0\%) e do Maranhão (61,4\%), que apresentaram elevados percentuais de consultório exclusivo para o Nasf. Com resultados também insatisfatórios, o estado de Roraima informou não ter disponibilidade de veículo para sua atuação, e no Espírito Santo, somente uma equipe informou ter veículo disponível (tabela 2).

Apesar dos resultados positivos e dos recursos investidos na $\mathrm{AB}$ advindos do PMAQ e do Programa de requalificação das Unidades Básicas de Saúde/Requalifica UBS, ainda há inadequação ou insuficiência de estrutura e insumos nas UBS, principalmente nos estados do Norte e do Nordeste, possivelmente associados ao baixo desenvolvimento econômico e oferta de serviços. Em contraposição, as regiões Sul e Sudeste, mais desenvolvidas e com maior número de serviços, apresentam resultados mais satisfatórios quanto à infraestrutura ${ }^{25-28}$. Ressalta-se, ainda, que as UBS cujas equipes aderiram ao PMAQ, e que, portanto, foram objeto de avaliação por parte das equipes Nasf, são aquelas que apresentam melhores condições estruturais, inclusive com equipes completas ${ }^{29,30}$.

Resultados não tão satisfatórios foram observados em relação à oferta de formação específica (por ocasião do início do trabalho) e educação permanente (processo contínuo de revisão das práticas de trabalho), em que, respectivamente, $45,6 \%$ e $51,6 \%$ das equipes informaram ter tido as referidas ações de formação para todos os profissionais da equipe (tabela 2).

Dentre os temas mais abordados na educação permanente, destacaram-se a organização do processo de trabalho do Nasf $(83,8 \%)$, saúde mental (80,7\%), conflitos sociais (79,7\%), princípios e diretrizes da $\mathrm{AB}$ (76,1\%), organização do processo de trabalho da $\mathrm{AB}(75,3 \%)$ e condições crônicas $(74,8 \%)^{\mathbf{3 0}}$.

É importante assinalar que a formação específica e a educação permanente foram referidas como sendo ofertadas para alguns ou para nenhum profissional por $54,4 \%$ e $48,4 \%$ das equipes respectivamente. Os resultados foram ainda piores nos estados de Rondônia e de Roraima, onde a totalidade das equipes registrou a inexistência de qualquer tipo de oferta de capacitação. No Espírito Santo, nenhuma equipe informou ter recebido educação permanente (tabela 2). Tais resultados contrastam com os observados no $1^{\circ}$ ciclo do PMAQ destinado exclusivamente às equipes $\mathrm{AB}$, em que mais de $80,0 \%$ das equipes informaram inciativas de educação permanente, ainda que em municípios de pequeno porte o percentual tenha sido menor $(67,4 \%)^{32}$.

Estudos anteriores já apontavam deficiência na formação, falta de apoio para capacitação e qualificação dos profissionais e necessidade de educação permanente 16,33-35. Tendo em consideração que as ações de educação permanente se embasam nos problemas cotidianos e no protagonismo dos profissionais, investir nessas ações pode contribuir para o fortalecimento e comprometimento dos profissionais, assim como motivá-los e mantê-los nos serviços de saúde 32,36,37.

\section{Operacionalização do trabalho entre as equipes Nasf e AB}

Considerando a gestão das equipes Nasf, a existência de coordenação foi referida por $92,6 \%$ das equipes. Resultado positivo foi observado em quase todos os estados, com mais de $75,0 \%$ das equipes informando a existência de coordenação, exceto Mato Grosso (64,7\%). Destaca-se, entretanto, o registro de equipes sem coordenação em quase todos os estados, exceção observada nos estados de Roraima, do Espírito Santo e no Distrito Federal, nos quais todas as equipes registraram haver coordenação (tabela 3). 
Tabela 3. Coordenação da equipe Nasf, segundo Grandes Regiões e Unidades da Federação, 2014

\begin{tabular}{|c|c|c|c|c|c|c|}
\hline \multirow{3}{*}{$\begin{array}{l}\text { Grandes Regiões e Unida- } \\
\text { des da Federação }\end{array}$} & \multirow{2}{*}{\multicolumn{2}{|c|}{$\begin{array}{l}\text { Existência de coordenação } \\
\qquad(\mathrm{N}=1.774)\end{array}$}} & \multicolumn{4}{|c|}{$\begin{array}{l}\text { Instância que realiza a coordenação } \\
\qquad(\mathrm{N}=1.642)\end{array}$} \\
\hline & & & \multicolumn{2}{|c|}{ Comum com AB } & \multicolumn{2}{|c|}{ Específica Nasf } \\
\hline & $\mathbf{N}$ & $\%$ & $\mathbf{N}$ & $\%$ & $\mathrm{~N}$ & $\%$ \\
\hline Brasil & 1.642 & 92,6 & 444 & 27,0 & 1.012 & 61,6 \\
\hline Norte & 111 & 88,1 & 26 & 23,4 & 80 & 72 \\
\hline Rondônia & 7 & 77,8 & 4 & 57,1 & 3 & 42,9 \\
\hline Acre & 7 & 77,8 & - & - & 7 & 100,0 \\
\hline Amazonas & 24 & 82,8 & 6 & 25,0 & 16 & 66,7 \\
\hline Roraima & 4 & 100,0 & 1 & 25,0 & 3 & 75,0 \\
\hline Pará & 35 & 92,1 & 7 & 20,0 & 27 & 77, \\
\hline Amapá & 15 & 88,2 & 1 & 6,7 & 14 & 93,3 \\
\hline Tocantins & 19 & 95,0 & 7 & 36,8 & 10 & 52,6 \\
\hline Nordeste & 768 & 93,5 & 204 & 26,6 & 506 & 65,5 \\
\hline Maranhão & 42 & 95,5 & 6 & 14,3 & 34 & 81,0 \\
\hline Piauí & 78 & 90,7 & 18 & 23,1 & 55 & 70,5 \\
\hline Ceará & 137 & 97,2 & 29 & 21,2 & 103 & 75,2 \\
\hline Rio Grande do Norte & 54 & 94,7 & 16 & 29,6 & 31 & 57,4 \\
\hline Paraíba & 109 & 94,0 & 28 & 25,7 & 63 & 57,8 \\
\hline Pernambuco & 136 & 96,5 & 36 & 26,5 & 92 & 67,6 \\
\hline Alagoas & 47 & 87,0 & 8 & 17,0 & 36 & 76,6 \\
\hline Sergipe & 11 & 91,7 & 3 & 27,3 & 8 & 72,7 \\
\hline Bahia & 154 & 90,6 & 60 & 39,0 & 84 & 54,5 \\
\hline Sudeste & 489 & 93,9 & 136 & 27,8 & 271 & 55,4 \\
\hline Minas Gerais & 275 & 93,2 & 89 & 32,4 & 139 & 50,5 \\
\hline Espírito Santo & 5 & 100,0 & 3 & 60,0 & 2 & 40,0 \\
\hline Rio de Janeiro & 63 & 88,7 & 19 & 30,2 & 36 & 57, \\
\hline São Paulo & 146 & 97,3 & 25 & 17,1 & 94 & 64,4 \\
\hline Sul & 175 & 92,6 & 43 & 24,6 & 104 & 59,4 \\
\hline Paraná & 72 & 94,7 & 17 & 23,6 & 48 & 66,7 \\
\hline Santa Catarina & 78 & 95,1 & 16 & 20,5 & 45 & 57,7 \\
\hline Rio Grande do Sul & 25 & 80,6 & 10 & 40,0 & 11 & 44,0 \\
\hline Centro-Oeste & 99 & 84,6 & 35 & 35,4 & 51 & 51,5 \\
\hline Mato Grosso do Sul & 33 & 86,8 & 8 & 24,2 & 20 & 60,6 \\
\hline Mato Grosso & 11 & 64,7 & 5 & 45,5 & 3 & 27,3 \\
\hline Goiás & 53 & 88,3 & 21 & 39,6 & 27 & 50,5 \\
\hline Distrito Federal & 2 & 100,0 & 1 & 50,0 & 1 & 50,0 \\
\hline
\end{tabular}

Fonte: Elaboração própria, com base em dados do Banco de dados do PMAQ, segundo ciclo, 2014.

Nota: Dado numérico igual a zero não resultante de arredondamento. 
Identificando-se a instância responsável pela coordenação, observou-se que apenas $27,0 \%$ das equipes informaram haver uma coordenação comum para as equipes Nasf e AB, qual seja, a coordenação da AB. Predominou a coordenação específica para o Nasf, informada por $61,6 \%$ das equipes. Este foi o padrão para todos os estados, exceto Rondônia, Espírito Santo e Mato Grosso, nos quais predominou a coordenação comum ou AB. No Distrito Federal, a coordenação foi referida igualmente entre a específica e a comum (tabela 3).

Embora a coordenação comum entre as equipes $\mathrm{AB}$ e Nasf, por si só, não assegure a integração entre as equipes, sua existência pode ser um fator facilitador. Argumentase que a existência de espaços coletivos e de cogestão, em que objetivos e estratégias de atuação sejam compartilhados, podem potencializar a institucionalização do Nasf, o apoio matricial e a integração entre $\mathrm{AB}$ e Nasf ${ }^{38}$.

Em relação às ações realizadas por meio do apoio matricial, o planejamento de atividades realizado de forma conjunta entre as equipes Nasf e AB foi relatado por $87,0 \%$ das equipes Nasf (tabela 4). Corroborando esse resultado, a pactuação das atividades da agenda de trabalho, entre as duas equipes, foi informada por $92,6 \%$ das equipes. O único estado com percentual de pactuação menor que 70,0\% foi Rondônia (55,6\%) (tabela 4).

Tabela 4. Apoio matricial - planejamento e avaliação realizados de forma conjunta entre equipes Nasf e AB, segundo Grandes Regiões e Unidades da Federação, 2014

\begin{tabular}{|c|c|c|c|c|c|c|c|c|c|c|c|c|c|c|c|c|}
\hline \multirow[t]{2}{*}{$\begin{array}{l}\text { Grandes Regiões } \\
\text { e Unidades da } \\
\text { Federação }\end{array}$} & \multirow{2}{*}{\multicolumn{2}{|c|}{$\begin{array}{c}\text { Planejamento } \\
(\mathrm{N}=1.706)\end{array}$}} & \multicolumn{2}{|c|}{$\begin{array}{l}\text { Pactuação do } \\
\text { cronograma de } \\
\text { atividades } \\
(\mathrm{N}=1.774)\end{array}$} & \multicolumn{2}{|c|}{$\begin{array}{l}\text { Ações não progra- } \\
\text { madas, em situa- } \\
\text { ções imprevistas } \\
\text { (sempre/na maio- } \\
\text { ria das vezes) } \\
(\mathrm{N}=1.774)\end{array}$} & \multicolumn{2}{|c|}{$\begin{array}{l}\text { Registro de } \\
\text { atividades } \\
\text { em prontuá- } \\
\text { rio comum } \\
(\mathrm{N}=1.774)\end{array}$} & \multicolumn{2}{|c|}{$\begin{array}{l}\text { Monitoramen- } \\
\text { to e análise de } \\
\text { indicadores e } \\
\text { informações } \\
\text { em saúde } \\
(\mathrm{N}=1.774)\end{array}$} & \multicolumn{2}{|c|}{$\begin{array}{c}\text { Monitora- } \\
\text { mento Projeto } \\
\text { Terapêutico } \\
\text { Singular } \\
\text { (PTS) } \\
\text { (N=1.774) }\end{array}$} & \multicolumn{2}{|c|}{$\begin{array}{l}\text { Monitoramento } \\
\text { e avaliação de } \\
\text { resultados da } \\
\text { atenção com- } \\
\text { partilhada } \\
(\mathrm{N}=1.774)\end{array}$} & \multicolumn{2}{|c|}{$\begin{array}{l}\text { Sem periodici- } \\
\text { dade definida } \\
\text { ou Periodici- } \\
\text { dade diferente } \\
\text { entre equipes } \\
(N=1.774)\end{array}$} \\
\hline & & & $\bar{N}$ & $\%$ & $\mathbf{N}$ & $\%$ & $\mathrm{~N}$ & $\%$ & $\mathrm{~N}$ & $\%$ & $\mathbf{N}$ & $\%$ & $\mathbf{N}$ & $\%$ & $\mathrm{~N}$ & $\%$ \\
\hline Norte & 88 & 79,3 & 107 & 84,9 & 102 & 81,0 & 100 & 79,4 & 80 & 63,5 & 68 & 54,0 & 64 & 50,8 & 46 & 36,5 \\
\hline Rondônia & 2 & 33,3 & 5 & 55,6 & 9 & 100,0 & 3 & 33,3 & 2 & 22,2 & 3 & 33,3 & 2 & 22,2 & 7 & 77,8 \\
\hline Acre & 6 & 85,7 & 8 & 88,9 & 5 & 55,6 & 7 & 77,8 & 8 & 88,9 & 6 & 66,7 & 6 & 66,7 & 3 & 33,3 \\
\hline Amazonas & 20 & 80,0 & 21 & 72,4 & 24 & 82,8 & 26 & 89,7 & 17 & 58,6 & 13 & 44,8 & 16 & 55,2 & 10 & 34,5 \\
\hline Tocantins & 14 & 73,7 & 17 & 85,0 & 14 & 70,0 & 13 & 65,0 & 13 & 65,0 & 9 & 45,0 & 11 & 55,0 & 5 & 25,0 \\
\hline Nordeste & 693 & 87,1 & 762 & 92,8 & 637 & 77,6 & 647 & 78,8 & 557 & 67,8 & 482 & 58,7 & 509 & 62 & 253 & 30,8 \\
\hline Maranhão & 31 & 73,8 & 40 & 90,9 & 27 & 61,4 & 34 & 77,3 & 31 & 70,5 & 24 & 54,5 & 30 & 68,2 & 13 & 29,5 \\
\hline Piauí & 72 & 86,7 & 79 & 91,9 & 64 & 74,4 & 54 & 62,8 & 60 & 69,8 & 52 & 60,5 & 48 & 55,8 & 27 & 31,4 \\
\hline Ceará & 128 & 92,8 & 130 & 92,2 & 108 & 76,6 & 120 & 85,1 & 99 & 70,2 & 87 & 61,7 & 78 & 55,3 & 38 & 27,0 \\
\hline $\begin{array}{l}\text { Rio Grande do } \\
\text { Norte }\end{array}$ & 53 & 96,4 & 55 & 96,5 & 45 & 78,9 & 47 & 82,5 & 42 & 73,7 & 36 & 63,2 & 38 & 66,7 & 13 & 22,8 \\
\hline Paraíba & 96 & 85,7 & 105 & 90,5 & 90 & 77,6 & 74 & 63,8 & 75 & 64,7 & 48 & 41,4 & 73 & 62,9 & 42 & 36,2 \\
\hline Pernambuco & 120 & 87,6 & 135 & 95,7 & 123 & 87,2 & 127 & 90,1 & 95 & 67,4 & 100 & 70,9 & 95 & 67,4 & 37 & 26,2 \\
\hline
\end{tabular}


Tabela 4. (cont.)

\begin{tabular}{|c|c|c|c|c|c|c|c|c|c|c|c|c|c|c|c|c|}
\hline Sudeste & 454 & 90,1 & 499 & 95,8 & 449 & 86,2 & 473 & 90,8 & 341 & 65,5 & 398 & 76,4 & 364 & 69,9 & 93 & 17,9 \\
\hline Minas Gerais & 262 & 91,9 & 283 & 95,9 & 257 & 87,1 & 260 & 88,1 & 196 & 66,4 & 217 & 73,6 & 203 & 68,8 & 50 & 16,9 \\
\hline Espírito Santo & 3 & 75,0 & 4 & 80,0 & 2 & 40,0 & 4 & 80,0 & 4 & 80,0 & 2 & 40,0 & 3 & 60,0 & 3 & 60,0 \\
\hline Rio de Janeiro & 54 & 79,4 & 65 & 91,5 & 61 & 85,9 & 63 & 88,7 & 40 & 56,3 & 54 & 76,1 & 43 & 60,6 & 22 & 31,0 \\
\hline São Paulo & 135 & 91,8 & 147 & 98,0 & 129 & 86,0 & 146 & 97,3 & 101 & 67,3 & 125 & 83,3 & 115 & 76,7 & 18 & 12,0 \\
\hline Sul & 155 & 84,7 & 168 & 88,9 & 166 & 87,8 & 175 & 92,6 & 105 & 55,6 & 122 & 64,6 & 101 & 53,4 & 58 & 30,7 \\
\hline Paraná & 56 & 78,9 & 66 & 86,8 & 68 & 89,5 & 72 & 94,7 & 35 & 46,1 & 44 & 57,9 & 33 & 43,4 & 31 & 40,8 \\
\hline Santa Catarina & 74 & 90,2 & 75 & 91,5 & 77 & 93,9 & 76 & 92,7 & 51 & 62,2 & 58 & 70,7 & 50 & $61,0,0$ & 18 & 22,0 \\
\hline $\begin{array}{l}\text { Rio Grande do } \\
\text { Sul }\end{array}$ & 25 & 83,3 & 27 & 87,1 & 21 & 67,7 & 27 & 87,1 & 19 & 61,3 & 20 & 64,5 & 18 & 58,1 & 9 & 29,0 \\
\hline Centro-Oeste & 95 & 84,8 & 107 & 91,5 & 91 & 77,8 & 77 & 65,8 & 70 & 59,8 & 71 & 60,7 & 62 & 53,0 & 41 & 35,0 \\
\hline $\begin{array}{l}\text { Mato Grosso } \\
\text { do Sul }\end{array}$ & 33 & 91,7 & 34 & 89,5 & 29 & 76,3 & 27 & 71,1 & 27 & 71,1 & 22 & 57,9 & 20 & 52,6 & 11 & 28,9 \\
\hline Mato Grosso & 11 & 68,8 & 12 & 70,6 & 13 & 76,5 & 10 & 58,8 & 5 & 29,4 & 5 & 29,4 & 5 & 29,4 & 9 & 52,9 \\
\hline Goiás & 50 & 86,2 & 59 & 98,3 & 47 & 78,3 & 38 & 63,3 & 38 & 63,3 & 43 & 71,7 & 37 & 61,7 & 21 & 35,0 \\
\hline Distrito Federal & 1 & 50,0 & 2 & 100,0 & 2 & 100,0 & 2 & 100,0 & - & - & 1 & 50,0 & - & - & - & - \\
\hline
\end{tabular}

Fonte: Elaboração própria, com base em dados do Banco de dados PMAQ, segundo ciclo, 2014.

Nota: Dado numérico igual a zero não resultante de arredondamento.

Registra-se ainda que as equipes identificaram haver flexibilização para atuar nas situações não previstas. A possibilidade de realizar ações não programadas em situações imprevistas foi registrada por $81,5 \%$ das equipes Nasf, como sendo realizada sempre ou na maioria das vezes (tabela 4).

Em relação ao uso de prontuário comum entre as equipes Nasf e $\mathrm{AB}$, observou-se que $83,0 \%$ das equipes Nasf informaram positivamente. A exceção dos estados de Rondônia (33,3\%), do Piauí (62,8\%), da Paraíba (63,8\%), de Alagoas (63,8\%), de Sergipe (58,3\%) e de Goiás (63,3\%), nos outros estados, ao menos, $65,0 \%$ das equipes referiram seu uso (tabela 4 ).

Cabe ainda destacar o monitoramento e a análise de indicadores e informações em saúde realizada pelas equipes $\mathrm{AB}$ e Nasf referido por 65,0\% das equipes Nasf. Menos da metade das equipes Nasf informou realizá-los nos estados de Rondônia (22,2\%), de Mato Grosso (29,4\%) e do Paraná (46,1\%). Pior resultado ainda foi registrado no Distrito Federal, onde nenhuma equipe informou fazer monitoramento (tabela 4).
Ainda no campo do monitoramento e avaliação, resultado similar foi encontrado para o monitoramento conjunto pelas duas equipes do Projeto Terapêutico Singular (PTS), informado por $64,3 \%$ das equipes Nasf. Novamente ganharam destaque negativo os estados de Rondônia e de Mato Grosso com, respectivamente, apenas 33,3\% e $29,4 \%$ das equipes informando realizá-los. Acompanharam este resultado os estados do Amazonas, do Tocantins, de Alagoas, da Paraíba e do Espírito Santo, em que um pouco mais de $40,0 \%$ das equipes informaram o monitoramento do PTS (tabela 4).

Resultado semelhante foi assinalado para o monitoramento e avaliação de resultados da atenção compartilhada, referido por $62,0 \%$ das equipes Nasf. As equipes dos estados de Rondônia e de Mato Grosso informaram que apenas $22,2 \%$, e $29,4 \%$ delas, respectivamente, monitoravam e avaliavam a atenção compartilhada (tabela 4).

Esses resultados indicam fragilidade e necessidade de incrementar o monitoramento do PTS e da atenção compartilhada bem 
como a análise e monitoramento de indicadores em saúde. O monitoramento das ações desenvolvidas pelas equipes integra o planejamento e constitui componente estratégico para o processo de trabalho e mudança das práticas em saúde ${ }^{39,40}$.

Quanto à periodicidade de reuniões entre equipes Nasf e $A B$ para o desenvolvimento do apoio matricial, assinala-se que quase um terço das equipes Nasf (27,7\%) no País ainda referiu não haver periodicidade predefinida (sem periodicidade e periodicidade diferente entre as equipes). Resultados mais negativos foram encontrados nos estados de Rondônia, de Roraima, de Alagoas, do Espírito Santo e de Mato Grosso, em que 78,8\%, 50,0\%, 59,3\%, $60,0 \%$ e $52,9 \%$, respectivamente, das equipes Nasf fizeram tal registro (tabela 4).

Destacaram-se as atividades de apoio matricial, realização de visitas (92,8\%), discussão de casos clínicos mais complexos e de eventos sentinela $(88,8 \%)$, intervenção no território (86,5\%) e realização de consultas compartilhadas (83,5\%), como aquelas com maior potencial para incentivar a integração e atuação conjunta entre as equipes (tabela 5). Tais atividades são esperadas considerando-se as diretrizes ministeriais para sua atuação.

Tabela 5. Atividades do apoio matricial e registro de informação utilizado pela equipe Nasf, segundo Grandes Regiões e Unidades da Federação, 2014

\begin{tabular}{lrr}
\hline Variáveis & $\mathbf{N}$ & \% \\
\hline Atividades de apoio matricial realizadas nos encontros entre Nasf/AB (N=1.774) & & 92,8 \\
\hline Realizam visitas com os profissionais da sua equipe & 1.646 & 88,8 \\
Discussão de casos, eventos sentinelas, casos difíceis e desafiadores & 1.575 & 86,5 \\
Organizam intervenções no território em conjunto com sua equipe & 1.534 & 83,5 \\
Consultas compartilhadas com os profissionais da Equipe de Atenção Básica & 1.482 & \\
\hline Registro de informação (N=1.774) & 1.727 & 97,4 \\
\hline Sim & 47 & 2,6 \\
Não & 1.127 & 65,3 \\
\hline Dispositivo utilizado para registro de informações (N=1.727) & 763 & 44,2 \\
\hline Fichas, planilhas ou relatórios construídos pela própria equipe NASF & 538 & 31,2 \\
SIAB & 315 & 18,2 \\
Sistema próprio do município/equipe & 267 & 15,5 \\
e-SUS & 264 & 15,3 \\
Outros & & \\
SIA & & \\
\hline
\end{tabular}

Fonte: Elaboração própria, com base em dados do Banco de dados PMAQ, segundo ciclo, 2014.

Significativo percentual das equipes Nasf (97,4\%) referiu registro das ações realizadas em algum sistema de informação. Porém, apesar de o MS disponibilizar sistemas para registro das atividades na $\mathrm{AB}$ (Sistema de Informação Ambulatorial/SIA, Sistema de Informações da AB/e-SUS e Sistema de Informação da Atenção Básica/Siab), chamou a atenção que $65,3 \%$ das equipes informaram ter criado seus formulários, fichas ou relatórios para registro de suas ações (tabela 5). 
Esse resultado evidencia uma dispersão e possivelmente repetição de registro das informações entre os sistemas oficiais e entre os criados pelas equipes. A fragmentação e duplicidade de registro não são eficientes, não favorecem a integração entre as equipes Nasf e AB, tampouco a gestão, planejamento, monitoramento e avaliação das ações realizadas no âmbito da $\mathrm{AB}^{34,35,39,40}$.

\section{Considerações finais}

O estudo se destaca como sendo o primeiro diagnóstico, de âmbito nacional, sobre o trabalho que as equipes Nasf realizam com as equipes $\mathrm{AB}$, tendo em vista que a avaliação externa do PMAQ do $1^{\circ}$ ciclo não contemplou as equipes Nasf.

O estudo permitiu reconhecer os avanços da implantação do Nasf, assim como importantes desafios para que a $\mathrm{AB}$ seja fortalecida na sua capacidade de reconhecer necessidades em saúde e de responder de forma mais oportuna e adequada, seja no âmbito de sua intervenção, seja em outros pontos de atenção dentro do sistema de saúde e no diálogo com outras políticas intersetoriais.

Evidenciou ainda o avanço da política no sentido do fortalecimento da APS, com a incontestável implantação de equipes matriciadoras em todo o País, apesar do espaço que ainda existe para a sua expansão quantitativa. Ainda há equipes de $\mathrm{AB}$ em regiões brasileiras que, por não alcançarem as condições exigidas para adesão ao PMAQ, não foram contempladas com equipes Nasf e, portanto, não estão representadas no estudo.

Embora se reconheça a importância do incentivo financeiro dado pelo PMAQ aos municípios para implantarem as equipes ESF e Nasf, o mecanismo por si só não assegura que regiões prioritárias com populações mais vulneráveis recebam tais recursos e implantem equipes.

Apesar dos resultados satisfatórios encontrados, permanecem existindo algumas insuficiências que, possivelmente, comprometem a resolutividade e a qualidade do cuidado ofertado na AB. Ainda é problema o investimento na capacitação continuada e qualificação dos profissionais tanto nos aspectos assistenciais quanto no uso de dispositivos que propiciem mais integração entre as equipes Nasf e $\mathrm{AB}$, em especial o matriciamento. Entre eles, chamou a atenção de forma negativa o monitoramento do plano terapêutico, da atenção compartilhada e dos indicadores em saúde.

Além disso, alguns estados com resultados mais negativos em diferentes aspectos avaliados merecem atenção e ações diferenciadas por parte do MS e dos respectivos governos estaduais e municipais.

Os resultados apresentados não estão influenciados pela atual PNAB de 2017 e expressam o período de vigência da PNAB 2012, em que o matriciamento configurava-se como atividade central do Nasf. A atual PNAB parece expressar que a dimensão assistencial seja o núcleo central da atividade do Nasf, contudo é prematuro ainda tecer considerações sobre a atual PNAB tendo em vista que ainda está por ser operacionalizada.

$\mathrm{O}$ arranjo organizacional proposto pelo Nasf expressa-se por meio das interações estabelecidas entre os profissionais, no seu modo de operar e nas formas de colaboração. $\mathrm{O}$ fato das formações especificas dos profissionais da $A B$ e do Nasf não darem suporte a essas práticas compartilhadas de trabalho acresce complexidade à sua operacionalização. Embora se reconheça a dificuldade em captar e mensurar a colaboração entre profissionais, no âmbito assistencial e técnico-pedagógico, preconizado pelo apoio matricial, também se reconhece a importância de esforços nesse sentido; e a avaliação do PMAQ é um deles ${ }^{35}$.

O diagnóstico fornecido pelo PMAQ é ainda insuficiente para afirmar que, de fato, o matriciamento esteja sendo praticado de forma satisfatória e trazendo aportes efetivos à resolutividade da atenção básica. Esta 
é uma lacuna de investigação a merecer estudos mais detalhados e específicos.

\section{Colaboradores}

Brocardo D contribuiu substancialmente para a concepção, interpretação e análise dos dados e elaboração do rascunho.
Andrade CLT contribuiu para o planejamento e análise dos dados e para a aprovação da versão final do manuscrito. Fausto MCR contribuiu para a análise de dados, revisão crítica de conteúdo e aprovação da versão final do manuscrito. Lima SML contribuiu análise de dados, revisão crítica de conteúdo e aprovação da versão final do manuscrito.

\section{Referências}

1. Kringos DS, Boerma WG, Hutchinson A, et al. The breadth of primary care: a systematic literature review of its core dimensions. BMC Health Serv Res. 2010; 10:65-78.

2. Macinko J, Starfield B, Shi L. The contribution of primary care systems to health outcomes within Organization for Economic Cooperation and Development (OECD) countries, 1970- 1998. BMC Health Serv Res. 2003; 38(3):831-865.

3. Mendes EV. A APS no Brasil. In: Mendes EV, organizador. O cuidado das condições crônicas na atenção primária à saúde: o imperativo da consolidação da estratégia da saúde da família. Brasília, DF: Organização Pan-Americana da Saúde; 2012. p. 71-99.

4. Saltman R, Rico A, Boerma W. Primary care in the driver's seat. New York: Open University Press; 2006.

5. Andrade LOM, Barreto ICH, Bezerra RC. A Atenção Primária à Saúde e Estratégia Saúde da Família. In: Campos GWS, Minayo MC, Drumond MJ, et al. Tratado de Saúde Coletiva. São Paulo: Hucitec; 2006.

6. Viana ALD, Fausto MCR. Atenção Básica e Proteção Social: universalismo x focalismo e espaço não mercantil da assistência. In: Viana ALD, Elias PE, Ibañez N, organizadores. Proteção Social: dilemas e desafios. São Paulo: Hucitec; 2005. p. 150- 167.

7. Gérvas J. Atención Primaria, de la teoría a la práctica. Cad Saúde Pública. 2008; 24(supl.1):S24-S26.

8. Giovanella L. Atenção Primária à Saúde seletiva ou abrangente. Cad Saúde Pública. 2008; 24(supl.1):S7$-\mathrm{S} 20$.

9. Schäfer WL, Boerma WG, Kringos DS, et al. QUALICOPC, a multi-country study evaluating quality, costs and equity in primary care. BMC Fam Pract. 2011; 12(115):1-9.

10. Brasil. Ministério da Saúde. Secretaria de Atenção à Saúde. Departamento de Atenção Básica. Política Nacional de Atenção Básica. Brasília, DF: MS; 2012. 
11. Brasil. Ministério da Saúde. Secretaria de Atenção à Saúde. Departamento de Atenção Básica. Diretrizes do NASF: Núcleo de Apoio Saúde da Família. Brasília, DF: Ministério da Saúde; 2009.

12. Brasil. Ministério da Saúde. Portaria no 154 de 24 de janeiro de 2008. Cria os Núcleos de Apoio à Saúde da Família - NASF. Diário Oficial da União. 24 Jan 2008 [acesso em 2016 mar 28]. Disponível em: http://dab.saude.gov.br/docs/legislacao/portarial54_24_01_08.pdf.

13. Brasil. Ministério da Saúde. Secretaria de Atenção à Saúde. Departamento de Atenção Básica. Núcleo de Apoio à Saúde da Família. Brasília, DF: MS; 2014.

14. Panizzi M. Avaliação da implantação dos Núcleos de Apoio à Saúde da Família - NASF [tese]. Florianópolis: Universidade Federal de Santa Catarina; 2015. $172 \mathrm{p}$.

15. Pasquim HM, Arruda MSB. Núcleo de Apoio à Saúde da Família: revisão narrativa sobre o apoio matricial na Atenção Básica. Corpus et Scientia. 2013 jul-dez; 9(2):34-44.

16. Patrocínio SSSM, Machado CV, Fausto MCR. Núcleo de Apoio à Saúde da Família: proposta nacional e implementação em municípios do Rio de Janeiro. Saúde debate. 2015; 39(esp):105-119.

17. Almeida ER. A Gênese dos Núcleos de Apoio à Saúde da Família [tese]. Salvador: Universidade Federal da Bahia; 2016. 215 p.

18. Sampaio J, Sousa CSM, Marcolino EC, et al. O NASF como dispositivo da gestão: limites e possibilidades. Rev Bras Ciênc Saúde. 2012; 6(3):317-324.

19. Brasil. Ministério da Saúde. Departamento de Atenção Básica. Banco de dados do PMAQ 2o Ciclo. Brasília, DF: MS; c2018 [acesso em 27 junho 2017]. Disponível em: http://dab.saude.gov.br/portaldab/ ape_pmaq.php?conteudo=2_ciclo.

20. Brasil. Ministério da Saúde. Secretaria de Atenção à Saúde. Departamento de Atenção Básica. Brasília,
DF: MS e IBGE. [acesso em 08 junho de 2017]. Disponível em: http://dab.saude.gov.br/dab/historico_ cobertura_sf/historico_cobertura_sf_relatorio.php.

21. Brasil. Ministério da Saúde. Secretaria de Atenção à Saúde. Departamento de Atenção Básica. Programa Nacional de Melhoria do Acesso e da Qualidade da Atenção Básica (PMAQ): manual instrutivo. Brasília, DF: MS; 2011.

22. Brasil. Ministério da Saúde. Secretaria de Atenção à Saúde. Departamento de Atenção à saúde. Programa Nacional de Melhoria do Acesso e da Qualidade da Atenção Básica (PMAQ-AB): Manual Instrutivo para Equipes de Atenção Básica (Saúde da Família, Saúde Bucal e Equipes Parametrizadas) e NASF. Brasília, DF: MS; 2013.

23. Viana AL, Machado CV. Proteção Social em Saúde: um balanço dos 20 anos do SUS. Physis. 2008; 18(4):645-684.

24. Brasil. Ministério da Saúde. Portaria no 3124 de 28 de dezembro de 2012. Redefine os parâmetros de vinculação dos Núcleos de Apoio à Saúde da Família (NASF) Modalidades 1 e 2 às Equipes Saúde da Família e/ou Equipes de Atenção Básica para populações específicas, cria a Modalidade NASF 3, e dá outras providências. Diário Oficial da União. 28 Dez 2012 [acesso em 2016 mar 28]. Disponível em: http://bvsms.saude.gov.br/bvs/saudelegis/ gm./2012/ portaria3124_28_12_2012.html.

25. Bousquat A, Giovanella L, Campos EMS, et al. Atenção primária à saúde e coordenação do cuidado nas regiões de saúde: perspectiva de gestores e usuários. Ciênc Saúde Colet. 2017; 22(4):1141-1154.

26. Oliveira IF, Amorim KMO, Paiva RA, et al. A Atuação do Psicólogo nos NASF: Desafios e Perspectivas na Atenção Básica. Temas Psicol. 2017 mar; 25(1):291-304.

27. Ribeiro MDA, Bezerra EMA, Costa MS, et al. Avaliação da atuação do núcleo de apoio à saúde da família. Ver Bras Promoção Saúde. 2014; 27(2):224231. 
28. Vannucchi AMC, Carneiro Junior N. Modelos tecnoassistenciais e atuação do psiquiatra no campo da atenção primária à saúde no contexto atual do Sistema Único de Saúde, Brasil. Physis. 2012; 22(3):963982.

29. Giovanella L, Mendonça MHM, Fausto MCR, et al. A provisão emergencial de médicos pelo Programa Mais Médicos e a qualidade da estrutura das unidades básicas de saúde. Ciênc Saúde Colet. 2016 set; 21(9):2697-2708.

30. Soares Neto JJ, Machado MH, Alves CB. O Programa Mais Médicos, a infraestrutura das Unidades Básicas de Saúde e o Índice de Desenvolvimento Humano Municipal. Ciênc Saúde Colet. 2016 set; 21(9):2709-2718.

31. Brocardo D. O Núcleo de Apoio à Saúde da Família/ NASF e Sua Interface com Atenção Básica: estudo a partir dos dados do Programa de Melhoria do Acesso e Qualidade da Atenção Básica/ PMAQ [dissertação]. Rio de Janeiro: Fiocruz/Ensp; 2018. 146 p.

32. Seidl H, Vieira SP, Fausto M, et al. Gestão do trabalho na atenção Básica em Saúde: uma análise a partir da perspectiva das equipes participantes do PMAQ-AB. Saúde debate. 2014; 38(esp):94-108.

33. Andrade LMB, Quandt FL, Campos DA, et al. Análise da implantação dos Núcleos de Apoio à Saúde da Família no interior de Santa Catarina. Saúde Transf Soc. 2012; 3(1):18-31.

34. Nascimento DDG, Oliveira MAC. Reflexões sobre as competências profissionais para o processo de trabalho nos Núcleos de Apoio à Saúde da Família.
Mundo Saúde. 2010; 34(1):92-96.

35. Silva ATCS, Aguiar ME, Winck K, et al. Núcleos de Apoio a Saúde da Família: desafios e potencialidade na visão dos profissionais da Atenção Primária do Município de São Paulo, Brasil. Cad Saúde Pública. 2012; 28(11):2076-2078.

36. Correia PCI, Goulart PM, Furtado JP. A avaliabilidade dos Núcleos de Apoio à Saúde da Família (Nasf). Saúde debate. 2017; 41(esp):345-359.

37. Rizzotto MLF, Gil CRR, Carvalho M, et al. Força de trabalho e gestão do trabalho em saúde: revelações da Avaliação Externa do Programa Nacional de Melhoria do Acesso e da Qualidade da Atenção Básica no Paraná. Saúde debate. 2014; 38(esp):237-251.

38. Panizzi M, Lacerda JT, Natal S, et al. Reestruturação produtiva na saúde: atuação e desafios do Núcleo de Apoio à Saúde da Família. Saúde debate. 2017; 41(112):155-170.

39. Silva E, Melo F, Sousa M, et al. Projeto Terapêutico Singular como Estratégia de Prática da Multiprofissionalidade nas Ações de Saúde. Rev Bras Ciênc Saúde. 2013 jun; 17(2):197-202.

40. Souza TS. Nasf: Fragmentação ou integração do trabalho na APS [dissertação]. Salvador: Universidade Federal da Bahia; 2015. p. 149

Recebido em: 30/05/2018

Aprovado em: 16/08/2018

Conflito de interesses: inexistente

Suporte financeiro: não houve 